\title{
ANÁLISIS DE UNA EXPERIENCIA DE DOCENCIA UNIVERSITARIA SEMIPRESENCIAL DESDE LA PERSPECTIVA DEL ALUMNADO
}

\author{
(ANALYSIS OF AN UNIVERSITY TEACHING EXPERIENCE OF BLENDED LEARNING SINCE \\ PERSPECTIVES'STUDENTS)
}

\author{
Manuel Area Moreira \\ Ana Luisa Sanabria Mesa \\ Miriam González Afonso \\ Universidad de La Laguna (España)
}

\section{RESUMEN}

En este artículo presentamos el análisis de las expectativas, valoraciones y opiniones manifestados por el alumnado perteneciente a dos grupos de la asignatura de Tecnología Educativa (Titulación de Pedagogía) de la Universidad de La Laguna, obtenidos al inicio y al final de un proceso de docencia apoyado en el uso continuado de un aula virtual y en sesiones presenciales de trabajo grupal y de tutorización. Esta experiencia de blended learning o docencia semipresencial se desarrolla en el marco institucional de la convocatoria de proyectos piloto para la docencia virtual convocado por el Vicerrectorado de Planes de Estudio y Títulos Propios correspondiente al curso 2006-07. El aula virtual de esta experiencia está alojada en el Campus Virtual de la Universidad de La Laguna (http://www.campusvirtual.ull.es) desarrollado bajo la plataforma Moodle. La recogida de datos de las perspectivas del alumnado se realizó en dos fases: una primera con un cuestionario inicial de expectativas al comienzo del curso, y otra final con otro cuestionario de valoración al finalizar la experiencia.

Palabras clave: campus virtual universitario, docencia virtual, blended learning o metodología semipresencial, metodología ECTS, tecnologías de la información y la comunicación, innovación y cambio educativo.

\section{ABSTRACT}

In this article we present the analysis of the expectations, appraisals and opinions declared by the alumnado belonging to two groups (TO and B) of the subject of Educational Technology (Qualification of Teaching) of the University of The Gap, obtained al start and al final of a process of teaching supported in the use continued of a virtual classroom and in eyewitness sessions of group work and of tutorización. This experience of blended learning or teaching semipresencial develops in the institutional framework of the assembly of pilot projects for the virtual teaching called by the Vicerrectorado of Plans of Study 
and corresponding Own Titles al course 2006-07. The virtual classroom of this experience is lodged in the Virtual Campus of the University (http://www.campusvirtual.ull.es) developed under the platform Moodle. The information collection of the perspectives of the students was realized in two phases: the first one with an initial questionnaire of expectations to the beginning of the course(year), and another final with another questionnaire on having finished the experience.

Key words: virtual university campus, virtual teaching, blended learning or methodology semipresencial, methodology ECTS, technologies of the information and the communication, innovation and educational change.

La docencia universitaria desde hace algunos años se encuentra en un período de reflexión y búsqueda de nuevas alternativas pedagógicas que le permitan innovarse y hacer frente a las nuevas exigencias que impone la denominada sociedad de la información, y específicamente, la incorporación y uso de las tecnologías digitales a la enseñanza. Existe abundante bibliografía al respecto (Colas y De Pablos, 2005; Fonseca y Aguaded, 2007; Cebrian, 2003; Sangrá y Glez, 2004).

La creación de campus virtuales para la docencia universitaria y específicamente de aulas virtuales que posibilitan el desarrollo de procesos de enseñanza aprendizaje mediante la utilización de las redes y recursos que ofrece Internet, está propiciando que en numerosas universidades tradicionales basadas en una educación presencial empiecen a ponerse en práctica experiencias de docencia que combinan actividades desarrolladas en las aulas físicas con otras a través delas aulas virtuales. Esta modalidad educativa recibe el nombre de blended learning o enseñanza semipresencial (Boyle y otros, 2003; Garrison y Kanuka, 2004; Vignare y otros, 2005).

Este artículo describe y analiza una experiencia de docencia virtual apoyada en la metodología "blended learning" desarrollada en una universidad presencial. Para ello se ha estructurado este trabajo en dos partes: En la primera se ofrece una descripción general del aula virtual (interfacey la estructura organizativa) explicitándose asimismo los objetivos y principios pedagógicos del método de enseñanza desarrollado en esta experiencia semipresencial. En la segunda parte se presentan los resultados del análisis de los puntos de vista del alumnado participante recogidos a través de dos encuestas, una inicial y otra final. La cumplimentación de las mismas se planteó de forma integrada como una actividad más que tenía que ser desarrollada dentro del entorno del aula virtual. A través de dichos cuestionarios se obtuvieron datos relativos al uso de Internet por parte del alumnado, a las expectativas iniciales que tienen en torno a los contenidos de la materia y del aula virtual, así como a la valoración final que hacen de los distintos aspectos de la experiencia y de utilidad de las TIC's en la formación universitaria. 


\section{DESCRIPCIÓN DE UNA EXPERIENCIA DE DOCENCIA SEMIPRESENCIAL A TRAVÉS DE UN AULA VIRTUAL MOODLE: EL CASO DE LA ASIGNATURA DE "TECNOLOGÍA EDUCATIVA" EN LA UNIVERSIDAD DE LA LAGUNA}

\section{El contexto institucional}

Desde el curso 2005-06 el Vicerrectorado de Planes de Estudio y Títulos Propios la Universidad de La Laguna (Islas Canarias-España) está realizando distintas convocatorias al profesorado de dicha universidad para la realización de Proyectos Piloto de experimentación de la llamada "docencia virtual". En dicha convocatoria se establecía que el principal objetivo era que el profesorado experimentase la puesta en práctica de nuevos métodos educativos apoyados en el uso de las TIC's y que estuvieran vinculados, en la medida de lo posible, con la metodología ECTS. La selección y evaluación de dichos proyectos fue delegada en una Unidad de Docencia Virtual integrada en el organigrama del citado Vicerrectorado que también tenía el cometido de formar y apoyar al profesorado en su ejecución. Por otra parte, el soporte tecnológico a dichos proyectos fue asumido por la FUE (Fundación Universidad-Empresa) empleándose los servidores alojados en la misma administrados por los técnicos informáticos de dicha Fundación. El software o plataforma de teleformación utilizada fue MOODLE, creándose un espacio telemático denominado Campus Virtual de la ULL en la siguiente dirección http://www.campusvirtual.ull.es ${ }^{1}$.

Ante dicha convocatoria el profesorado que impartía la asignatura de la materia troncal de Tecnología Educativa de la titulación de Pedagogía perteneciente a la Facultad de Educación presentó un proyecto a dicha convocatoria, el cual fue aprobado por dicho Vicerrectorado tanto en el curso 2005-06, como 2006-07. Los datos que presentamos y se analizan en esta comunicación corresponden a la experiencia desarrollada en el segundo cuatrimestre del curso 2006-07 (entre los meses de febrero y junio) en el que participaron los alumnos y alumnas matriculados

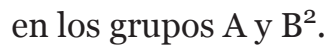

\section{Antecedentes docentes de la experiencia}

La utilización de la WWW para la impartición de esta materia empezó en esta universidad en el curso 1997-98. Desde entonces hasta la actualidad los recursos web utilizados han ido evolucionando adaptándose a las necesidades docentes de la asignatura. Una descripción de estos recursos durante el periodo 1997-2004 puede 
verse en Area y otros (2004). En el curso 2004-05 se produjo un salto cualitativo muy relevante en la metodológica desarrollada en esta asignatura respecto a cursos anteriores ya que se empezó a desarrollar una metodología semi-presencial de enseñanza (blended-learning) que combinara distintas actividades docentes desarrolladas a través de un aula virtual específicamente creada para esta asignatura, y sesiones presenciales en la clase habitual. Esta aula virtual docente se gestionó bajo la plataforma MOODLE 1.4.1 para el seguimiento y apoyo a las actividades tanto teóricas como prácticas. Esta plataforma estaba ubicada en el servidor de EDULLAB (Laboratorio de Educación y Nuevas Tecnologías) http://www.edullab.net, y sirvió como primer referente experiencial para el diseño y desarrollo del aula virtual que desarrollamos en el presente curso 2006-07.

\section{Los principios o supuestos psicodidácticos}

Los principios pedagógicos que inspiraron la metodología de enseñanza de esta experiencia fueron:

- Que el alumnado desarrollase un proceso de aprendizaje autónomo a partir de distintas tareas/actividades propuestas con sus correspondientes orientaciones de trabajo.

- Que el horario de estudio y dedicación a la asignatura fuera flexible sin las limitaciones y rigideces de los horarios de clase tradicionales de forma que se pudieran combinar actividades de tipo presencial con otras virtuales.

- Que el alumnado tuviera a su disposición distintos tipos de materiales didácticos en formatos diversos: bibliografía impresa, documentos electrónicos en Internet que le permitieran el desarrollo de su propio proceso de aprendizaje.

- Que se le plantearan problemas/tópicos sobre los que éste debiera reflexionar críticamente y buscara soluciones a los mismos bien en forma de ensayo, bien como trabajo de investigación.

- Que la labor y papel del profesor no consistiera en la transmisión expositiva de los contenidos, sino en la elaboración de materiales didácticos, en la supervisión y atención personalizada (tutorización) a los alumnos durante el proceso de desarrollo de trabajo de los mismos, así como en la evaluación de las tareas cumplimentadas por éstos.

- Que el alumnado desarrollara las competencias y habilidades de uso de las tecnologías de información y comunicación para el trabajo académico e 
intelectual propio de esta asignatura: buscar información en Internet, analizarla, reconstruirla críticamente y comunicarla.

- Que se estimulara la participación, debate e intercambio de ideas tanto entre alumnado como entre éstos y el profesor a través de espacios virtuales de comunicación como los foros y tablones de noticias.

- En definitiva, que la metodología de enseñanza asumiera los principios pedagógicos derivados del modelo ECTS que inciden en que el alumnado aprenda por sí mismo a través de un proceso de aprendizaje autónomo basado en la actividad.

\section{Descripción del aula virtual de la asignatura (2006-07)}

El diseño de la estructura y componentes didácticos de la misma fue realizado teniendo en cuenta por una parte, las posibilidades técnicas que permite el aula Moodle (las cuales son muchas y dan un margen de opcionalidad al docente amplio) junto con los principios psicopedagógicos que acabamos de citar. En síntesis los elementos y componentes incorporados a dicha aula fueron:

- Programa oficial de la asignatura.

- Chat de comunicación entre estudiantes.

- Foto de debate de la clase (abierto a alumnado y profesor).

- Noticias del profesor (tablón de anuncios).

- Cuestionario on line inicial (para identificar expectativas del alumnado al inicio del curso).

- Cuestionario on line final (para identificar valoraciones del alumnado sobre la asignatura al final del cuatrimestre).

- Libro de texto en PDF con una síntesis de los contenidos teóricos de la asignatura (5 temas más bibliografía).

- 3 prácticas individuales obligatorias a realizar a través del aula virtual.

- 1 práctica individual optativa de un curso sobre búsqueda de recursos de información en Internet (en colaboración con la Biblioteca Universitaria).

- 1 trabajo o proyecto de investigación a realizar grupalmente. 
- Cuestionarios en formato test de autoevaluación sobre los contenidos teóricos de cada tema.

- Una selección de sitios web relevantes en Internet.

- Un calendario con las fechas de las tareas a desarrollar a lo largo del cuatrimestre.

- Una sección de noticias de la Red de blogs y canales feed seleccionados.

\section{La metodología semipresencial desarrollada}

La metodología desarrollada se basó en la combinación de actividades presenciales con otras desarrolladas a través del aula virtual intentando que el proceso de aprendizaje se basara en la cumplimentación secuenciada de distintas tareas de forma autónoma bajo la supervisión del profesor, tal como se deriva de los planteamientos ECTS. En el grupo A la distribución del tiempo entre actividad presencial (a través de sesiones de clase y tutorías) fue del 50\% y virtual (50\%). En el grupo B esta distribución fue $70 \%$ presencial frente al $30 \%$ virtual.

Brevemente el proceso de trabajo a lo largo del cuatrimestre se desarrolló del siguiente modo:

- Fase inicial. Se desarrolló presencialmente en la sala de informática de la Facultad y consistió en llevar al alumnado en pequeños grupos para que éstos se inscribieran en el aula virtual, conocieran sus características y funcionamiento, y desarrollaran las primeras actividades de uso del foro, y contestaran al cuestionario on line inicial.

- Fase de desarrollo

- Sesiones de actividad presencial. Sesiones presenciales semanales desarrolladas una vez a la semana en el aula de clase con la finalidad de presentar y discutir los contenidos teóricos, y también para la exposición de los proyectos de investigación del alumnado. En el grupo B, también se incorporaron sesiones presenciales en el aula de informática.

- Sesiones de trabajo virtual. Se desarrolló de forma paralela y complementaria con las sesiones presenciales. Consistió en la cumplimentación de las tareas especificadas en el aula virtual, y la comunicación permanente a través del foro de la asignatura y el tablón de anuncios. 
- Acciones de tutorización y apoyo. El proceso de atención individualizada y/ o grupal para resolver dudas, aclaraciones o cualquier otra problemática de desarrollo tanto en el ámbito presencial del despacho en formato tutorías, como a través de los recursos informáticos de foro y correo electrónico.

- Fase evaluativa

- Evaluación continua de las prácticas individuales enviadas al aula virtual.

- Evaluación de los proyectos de investigación grupales. Esta evaluación fue realizada tanto por los propios alumnos (a través de un cuestionario on line ubicado dentro del aula virtual) como por el profesor.

- Evaluación de los contenidos teóricos a través de un examen presencial en formato test.

- Autoevaluación a través de cuestionario on line ofertados dentro del aula virtual.

\section{¿QUÉ OPINA EL ALUMNADO DE LA EXPERIENCIA DE ESTA AULA VIRTUAL? ANÁLISIS DE LOS CUESTIONARIOS INICIAL Y FINAL SOBRE EXPECTATIVAS Y VALORACIONES DEL ALUMNADO}

En esta segunda parte de la comunicación presentamos los resultados sobre las opiniones del alumnado acerca de la experiencia desarrollada en semipresencial o blended learning a través del aula virtual de la asignatura de Tecnología Educativa.

Con el fin de analizar la experiencia de docencia virtual desde la perspectiva del alumnado, nos planteamos los siguientes objetivos:

- Conocer el nivel de alfabetización tecnológica de los alumnos, sobre todo el nivel de uso de Internet.

- Conocer las expectativas sobre la asignatura de Tecnología Educativa.

- Conocer las expectativas sobre la metodología semipresencial y el uso del aula virtual.

- Analizar los cambios y evaluaciones producidas en las opiniones del alumnado, sobre los aspectos anteriormente señalados, antes de empezar la experiencia de docencia virtual y al finalizar la misma. 
Para la recogida de datos se diseñaron dos cuestionarios, uno inicial y otro final, que se plantearon como dos actividades integradas dentro del aula virtual, de forma que el alumnado los cumplimentaba en el propio entorno.

El cuestionario inicial se elaboró atendiendo a las tres dimensiones siguientes: alfabetización tecnológica sobre Internet, la segunda sobre experiencia en docencia virtual, y la tercera dimensión sobre asignatura de Tecnología Educativa. En todas, estaba formado por ocho ítems, siete de alternativa múltiple y uno de respuesta abierta. Cada dimensión esta compuesta de las siguientes subdimensiones:

- Alfabetización tecnológica sobre Internet: frecuencia de uso Internet, tipo de uso, y lugar de conexión.

- Experiencia en docencia virtual: experiencias previas y expectativas, y opinión sobre la docencia virtual.

- Asignatura de Tecnología Educativa: contenidos de la misma, y las expectativas y motivación inicial.

El cuestionario final se ha elaborado atendiendo a las dos últimas dimensiones del cuestionario inicial, incluyendo más subdimensiones en cada categoría. En total el cuestionario estaba formado por quince ítems, catorce de alternativa múltiple y uno de respuesta abierta. Las subdimensiones fueron:

- Experiencia de aula virtual: experiencia previa, valoración de la misma, lugar de conexión, utilidad de la experiencia, generalización de la misma.

- Asignatura de Tecnología Educativa: valoración de la asignatura, horas de trabajo, y valoración de la metodología semipresencial.

Los resultados del análisis los presentamos primero por cuestionario, y posteriormente analizaremos comparativamente los resultados obtenidos en el primer y segundo cuestionario.

\section{Resultados}

- Cuestionario inicial. Expectativas de los estudiantes hacia la asignatura:

El grupo A cuenta con 56 alumnos matriculados en la asignatura, y X en el aula virtual, cumplimentando el cuestionario 46 alumnos-as. El Grupo B tiene 56 alumnos 
matriculados en la asignatura, de los cuales 50 se han dado de alta en el aula virtual, y han cumplimentado el cuestionario 45 alumnos. Los resultados los presentamos atendiendo a cada una de las dimensiones y subdimensiones.

\section{Primera Dimensión: Alfabetización tecnológica en Internet:}

\begin{tabular}{|r|c|c|}
\hline \multicolumn{2}{|l}{ ¿Cuál es el grado de uso a Internet que realizas? } & \\
\hline & GRUPO A & GRUPO B \\
\hline Nunca (o casi nunca) he accedido a Internet: & 0 & $1(2,22 \%)$ \\
Muy pocas veces he usado Internet: & $1(2,17 \%)$ & $1(2,22 \%)$ \\
Más o menos una vez al mes: & $3(6,52 \%)$ & $5(11,11 \%)$ \\
Más o menos una vez a la semana: & $15(32,61 \%)$ & $12(26,67 \%)$ \\
Más o menos casi todos los días: & $27(58,70 \%)$ & $26(57,78 \%)$ \\
\hline
\end{tabular}

Tabla 1. Frecuencia de uso de Internet

\begin{tabular}{|r|c|c|}
\hline \multicolumn{2}{|l}{ ¿Cuál o cuáles de estos servicios/recursos de Internet utilizas? } \\
\hline Navegar por el WWW: & GRUPO A & GRUPO B \\
\hline Enviar y recibir correo electrónico: & $37(80,78 \%)$ & $38(84,44 \%)$ \\
Comunicarme a través del Messenger: & $42(91,30 \%)$ & $34(75,56 \%)$ \\
Elaborar mi propio blog y/o página web personal: & $6(13,04 \%)$ & $43(95,56 \%)$ \\
Bajar películas y/o música: & $30(65,22 \%)$ & $29(6,44 \%)$ \\
Jugar videojuegos en red: & $3(6,52 \%)$ & $7(15,56 \%)$ \\
Otros: & $6(13,04 \%)$ & $10(22,22 \%)$ \\
\hline
\end{tabular}

Tabla 2. Tipo de uso

\begin{tabular}{|r|c|c|}
\hline \multicolumn{2}{|l|}{ Habitualmente ¿desde dónde te conectas a Internet? } & \multicolumn{1}{l|}{} \\
\hline & GRUPO A & GRUPO B \\
\hline Desde mi casa: & $34(73,91 \%)$ & $29(64,44 \%)$ \\
Desde casa de un amigo/a: & $5(10,87 \%)$ & $5(11,11 \%)$ \\
Desde un cibercafé: & $4(8,70 \%)$ & $10(22,22 \%)$ \\
Desde un aula de la universidad: & $2(4,35 \%)$ & $2(4,44 \%)$ \\
No tengo un sitio fijo, sino que es variable: & $6(13,04 \%)$ & $7(15,56 \%)$ \\
No uso Internet: & 0 & 0 \\
\hline
\end{tabular}

Tabla 3. Lugar de conexión 
A partir de estos datos se puede decir que el alumnado de la asignatura de Tecnología Educativa se conecta casi todos los días y lo hace mayoritariamente desde su casa (ver tabla 1 y 3 respectivamente). El uso que hace de Internet es fundamentalmente comunicativo (uso de recursos para la comunicación), informativo (www) y para bajarse música y películas. Por lo tanto, podríamos definirlo como un usuario alfabetizado en el uso de Internet, utilizando tanto los recursos comunicativos como informativo (ver tabla 2). No existen diferencias significativas en esta dimensión entre el grupo de la mañana (Grupo A) y el grupo de la tarde (Grupo B).

\section{Segunda dimensión: Docencia virtual}

- Experiencia previa

\begin{tabular}{|c|c|c|c|}
\hline \multicolumn{4}{|l|}{ ¿Es la primera vez que utilizas un aula virtual para estudiar una asignatura? } \\
\hline & GRUPO A & GRUPO B \\
\hline Si: & $37(82,22 \%)$ & $40(88,89 \%)$ \\
No: & $8(17,78 \%)$ & $5(11,11 \%)$ \\
\hline
\end{tabular}

Tabla 4

- Opinión sobre la docencia virtual

\begin{tabular}{|c|c|c|}
\hline \multicolumn{3}{|l|}{ ¿Qué opinas de estudiar a través de un aula virtual? } \\
\hline & GRUPOA & GRUPO B \\
\hline Me parece bien porque da más flexibilidad y libertad al alumnado: & $35(76,09 \%)$ & $27(60,00 \%)$ \\
\hline $\begin{array}{r}\text { Me parece mal ya que estimula que los alumnos no vayamos a clase y no } \\
\text { nos impliquemos en la asignatura: }\end{array}$ & 0 & $1(2,22 \%)$ \\
\hline No me gusta porque no me atraen los ordenadores: & 0 & $1(2,22 \%)$ \\
\hline En esta asignatura me parece bien, pero no en las demás: & $5(10,87 \%)$ & $8(17,78 \%)$ \\
\hline Creo que debiera generalizarse para todas las asignaturas universitarias: & $7(15,22 \%)$ & $7(15,56 \%)$ \\
\hline $\begin{array}{r}\text { A través de un aula virtual se aprende más que a través de copiar } \\
\text { apuntes en clase: }\end{array}$ & $10(21,74 \%)$ & $4(8,89 \%)$ \\
\hline No tengo ninguna opinión formada: & $4(8,70 \%)$ & $8(17,78 \%)$ \\
\hline
\end{tabular}

Tabla 5

Los resultados señalan que para la mayoría del alumnado, ésta es su primera experiencia de enseñanza y aprendizaje virtual (ver tabla 4). A pesar de esto, su predisposición sobre la misma es positiva, como se puede observar con la tabla 5. Especialmente consideran que ésta favorecerá la flexibilidad y la libertad en el 
proceso de aprendizaje, además de considerar que se aprende más que en una clase presencial. Esta opinión se acentúa más en el Grupo A, donde un 21,74\% consideran que se aprenderá más que "a través de copiar apuntes en clase".

Resulta significativo que siendo más de las tres cuartas partes del alumnado (tabla 4) los que manifiestan que no ha cursado asignaturas donde se utilizara un aula virtual, y sin embargo consideren, al comienzo del curso, que este tipo de experiencia debe generalizarse al resto de las asignaturas (15,56\% en ambos grupos), aunque un $17,78 \%$ del alumnado del Grupo B opina que estudiar a través de una aula virtual es más propio de esta asignatura, frente a una 10,87\% del alumnado del Grupo A. Esto indica que los estudiantes tienen una predisposición positiva a este tipo de enseñanza y unas expectativas altas sobre las características y ventajas para el aprendizaje.

\section{Tercera dimensión: Asignatura de Tecnología Educativa}

- Contenidos de la asignatura

\begin{tabular}{|r|c|c|}
\hline Señala qué contenido/s son los propios o específicos de esta asignatura? & \\
\hline & GRUPO A & GRUPO B \\
\hline Materiales didácticos digitales: & $37(80,43 \%)$ & $39(86,67 \%)$ \\
E-learning: & $29(63,04 \%)$ & $19(42,22 \%)$ \\
Historia del curriculum técnico-racional: & $4(8,70 \%)$ & $1(2,22 \%)$ \\
Educación y Sociedad de la Información: & $24(52,17 \%)$ & $20(44,44 \%)$ \\
Historia de la Informática: & $4(8,70 \%)$ & $1(2,22 \%)$ \\
Psicología del aprendizaje: & $4(8,70 \%)$ & $1(2,22 \%)$ \\
Libros de texto: & $11(23,91 \%)$ & $2(4,44 \%)$ \\
Multimedia educativo: & $33(71,74 \%)$ & $31(68,89 \%)$ \\
Hardware y software: & $18(39,13 \%)$ & $16(35,56 \%)$ \\
Edublogs: & $6(13,04 \%)$ & $2(4,44 \%)$ \\
World Wide Web: & $6(13,04 \%)$ & $8(17,78 \%)$ \\
Teorías y métodos didácticos: & $16(34,78 \%)$ & $12(26,67 \%)$ \\
Organización escolar: & $5(10,87 \%)$ & $6(13,33 \%)$ \\
Software educativo: & $36(78,26 \%)$ & $30(66,67 \%)$ \\
\hline
\end{tabular}

Tabla 6 
- Expectativas y motivaciones sobre la asignatura

\begin{tabular}{|r|c|c|}
\hline ¿Qué expectativas o motivación tienes hacia esta asignatura? & \\
\hline & GRUPO A & GRUPO B \\
\hline Ninguna. No sé de que va: & $1(2,17 \%)$ & $2(4,44 \%)$ \\
No me gusta la tecnología, por lo que no me atrae esta asignatura: & 0 & $1(2,22 \%)$ \\
No espero ni mucho ni poco. Lo que quiero es aprobar: & 0 & $4(8,89 \%)$ \\
Tengo ilusión y ganas de saber cómo usar la tecnología en la educación: & $45(97,83 \%)$ & $38(84,44 \%)$ \\
\hline
\end{tabular}

Tabla 7

- Expectativas sobre el aprendizaje

Las respuestas del alumnado se clasificaron en tres categorías, que son: Conocer y aplicar las Tecnologías en la Educación, dominio en la alfabetización informática y conocimiento y experiencia de aula virtual. Estas tres categorías surgen a partir del análisis de las respuestas que da el alumnado a la pregunta sobre qué esperas (o te gustaría) aprender en esta asignatura. La categoría con más aportaciones fue la segunda: Dominio en la Alfabetización Informática. En casi todas las respuestas del alumnado, y con independencia de la categoría en las que se las clasifique, hay referencias a expectativas profesionales futuras.

Algunos ejemplos de las aportaciones del alumnado a cada una de estas categorías son las siguientes:

- Conocer y aplicar las Tecnologías en la Educación

- Aprender si a través de estos "nuevos" métodos de enseñanza podríamos llevar la educación a todos los lugares donde, por diversos motivos, no tienen o no pueden acceder a la educación.

- $\quad$ Aprender todo sobre las nuevas tecnologías en la educación y que me sirva para el terreno laboral.

- Me gusta aprender cosas nuevas...y esta asignatura aporta muchas de esas cosas...nos encontramos ante una sociedad que crece a pasos agigantados donde las tecnologías son cada vez más imprescindibles para la vida cotidiana... por lo que me resulta muy interesante como influye todo esto en la educación.

- Ampliar mis conocimientos sobre informática y sobre todo relacionarlo con la educación ya que es sobre lo que probablemente tenga relación nuestro futuro profesional. 
- Dominio en la alfabetización informática

- Aprender conocimientos básicos de informáticos para poder moverme por internet con libertad y poder crear paginas web.

- Dominar mejor lo que es internet, para así de esta forma ampliar mis conocimientos con la informática y sentirme mas capacitada en mi trabajo.

- Aprender a usar las nuevas tecnologías para que me ayuden en mi labor de pedagogo en un futuro.

- Mejorar las técnicas de búsqueda de información por internet, familiarizarme más con las nuevas tecnologías y avances.

- Conocimiento y experiencia de aula virtual

- Saber aplicar lo que aprendo en mi vida y ya sólo con entrar dentro del campus virtual me parece una idea genial.

- Aprender a trabajar de una forma novedosa para mi y soltarme en lo que se refiere a e-learning.

- Adquirir conocimientos sobre como poder aprender a través de un ordenador o Internet.

- Me gustaría aprender a estudiar mediante el ordenador, creo que ahorras tiempo y a la vez puede ser divertido y más ameno.

Los resultados de esta tercera dimensión indican que el alumnado considera que los contenidos propios de la asignatura de Tecnología Educativa se centran fundamentalmente en los materiales didácticos digitales, multimedia educativo y software educativos; no obstante, y sobre todo el grupo A, señalan también los contenidos sobre e-learning, y educación y sociedad de la información. También señalan, aunque en menor medida, los contenidos sobre hardware y software, y sobre teoría y métodos didácticos (ver tabla 6). Estos resultados parecen indicar que el alumnado tiene una idea clara de que en esta asignatura se va a trabajar todo lo concerniente a los materiales y medios en la educación. Otro elemento a destacar es que no existen diferencias significativas entre la opinión de Grupo A y el Grupo B, a pesar de que la docencia la desarrollan profesores diferentes en ambos grupos. Esto podría ser un indicador de la coordinación docente en la planificación de la asignatura y en la información inicial que se le proporciona al alumnado en la presentación de la asignatura. 
En cuanto a las expectativas y motivaciones hacia la asignatura, el alumnado de los dos grupos señala mayoritariamente que esperan aprender cómo usar las tecnologías en la educación (ver tabla 7). Respecto a las expectativas sobre el aprendizaje, la mayoría del alumnado espera conocer y aplicar las tecnologías en la educación; pero también alcanzar un mayor dominio en la alfabetización tecnológica. El ámbito sobre conocimiento y experiencia en el aula virtual, es señalado con mayor frecuencia por el alumnado del grupo B que por el del grupo A. En general, los estudiantes esperan adquirir conocimientos y competencias que puedan transferir posteriormente a su desarrollo profesional, tal y como se refleja en las opiniones que manifiestan en el ítem de preguntas abiertas del cuestionario.

\section{Cuestionario Final. Valoración de la experiencia por el alumnado}

El cuestionario final, tal y como hemos indicado, consta de quince ítems, de los cuales catorce son de respuestas múltiples y uno de respuesta abierta. Las dimensiones y subdimensiones utilizadas para su elaboración y análisis de los resultados, son las siguientes:

- Primera dimensión: Experiencia de aula virtual.

Subdimensiones: experiencia previa, lugar de conexión, valoración de la experiencia, utilidad y generalización de la misma.

- Segunda dimensión: Asignatura de Tecnología Educativa.

Subdimensiones: horas de trabajo, asistencia a las clases presenciales, valoración de la asignatura, del aula virtual y de la metodología semipresencial.

Del Grupo A, contestaron al cuestionario 28 alumnos, y del Grupo B, 32 alumnos de los 41 que han cursado la asignatura a través del aula virtual. Los resultados los presentamos por grupo y atendiendo a cada una de las dimensiones y subdimensiones.

Primera dimensión: Experiencia de aula virtual

\begin{tabular}{|c|c|c|}
\hline \multicolumn{3}{|c|}{ ¿Es la primera vez que estudias a través de un aula virtual? } \\
\hline & GRUPO A & GRUPO B \\
\hline SI: & $23(82,14 \%)$ & $30(93,75 \%)$ \\
NO: & $5(17,86 \%)$ & $2(6,25 \%)$ \\
\hline
\end{tabular}

Tabla 8. Experiencia previa 


\begin{tabular}{|r|c|c|}
\hline \multicolumn{3}{|c|}{ ¿Desde dónde te has estado conectando habitualmente al aula virtual de la asignatura? } \\
\hline & GRUPO A & GRUPO B \\
\hline Desde mi casa: & $20(71,43 \%)$ & $18(56,25 \%)$ \\
Desde la casa de un amigo o familiar: & $1(3,57 \%)$ & $2(6,25 \%)$ \\
Desde el aula de informática de la facultad: & $8(28,57 \%)$ & $7(21,88 \%)$ \\
Desde un cibercafé: & $4(14,29 \%)$ & $9(28,13 \%)$ \\
Desde el trabajo: & $1(3,57 \%)$ & 0 \\
Desde lugares diferentes. Variaba en cada momento: & $5(17,86 \%)$ & $8(25,00 \%)$ \\
\hline
\end{tabular}

Tabla 9. Lugar de Conexión

\begin{tabular}{|c|c|c|}
\hline \multicolumn{3}{|c|}{$\begin{array}{l}\text { Valora globalmente lo que ha sido esta experiencia de cursar la asignatura a través de esta aula } \\
\text { virtual }\end{array}$} \\
\hline & GRUPO A & GRUPO B \\
\hline $\begin{array}{r}\text { Muy satisfactoria. He aprendido mucho y me ha aportado cosas } \\
\text { nuevas: }\end{array}$ & $18(64,29 \%)$ & $19(59,38 \%)$ \\
\hline $\begin{array}{r}\text { Satisfactoria. Ha tenido algunos aspectos positivos, pero otros } \\
\text { no tanto: }\end{array}$ & $10(35,71 \%)$ & $13(40,63 \%)$ \\
\hline Indiferente. Me ha dado lo mismo: & 0 & 0 \\
\hline $\begin{array}{r}\text { Insatisfactoria. En conjunto no me ha gustado. Alguna cosa bien, } \\
\text { pero la mayoría mal: }\end{array}$ & 0 & 0 \\
\hline $\begin{array}{r}\text { Muy insatisfactoria. No me ha gustado nada. No quisiera volver } \\
\text { a repetirlo: }\end{array}$ & 0 & 0 \\
\hline
\end{tabular}

Tabla 10. Valoración de la experiencia

\begin{tabular}{|c|c|c|}
\hline \multicolumn{3}{|c|}{ ¿Qué opinión tienes de la utilidad de las aulas virtuales? (puedes elegir varias opciones) } \\
\hline & GRUPOA & GRUPO B \\
\hline $\begin{array}{r}\text { Creo que es una metodología necesaria para la mejora de la enseñanza } \\
\text { en la universidad: }\end{array}$ & $14(50,00 \%)$ & $22(68,75 \%)$ \\
\hline $\begin{array}{r}\text { Creo que es una metodología que está de moda, pero que no tendrá } \\
\text { éxito: }\end{array}$ & $2(7,14 \%)$ & 0 \\
\hline $\begin{array}{r}\text { Creo que en el futuro casi todas las asignaturas universitaria tendrán } \\
\text { aulas virtuales: }\end{array}$ & $14(50,00 \%)$ & $22(68,75 \%)$ \\
\hline $\begin{array}{r}\text { Creo que las aulas virtuales no sirven para mejorar la docencia ni el } \\
\text { aprendizaje: }\end{array}$ & 0 & 0 \\
\hline Creo que es una metodología útil para el aprendizaje de los estudiantes: & $12(42,86 \%)$ & $17(53,13 \%)$ \\
\hline Creo que en unos casos funciona y en otros no. Depende del profesor: & $9(32,14 \%)$ & $13(40,63 \%)$ \\
\hline
\end{tabular}

Tabla 11. Utilidad de la experiencia 


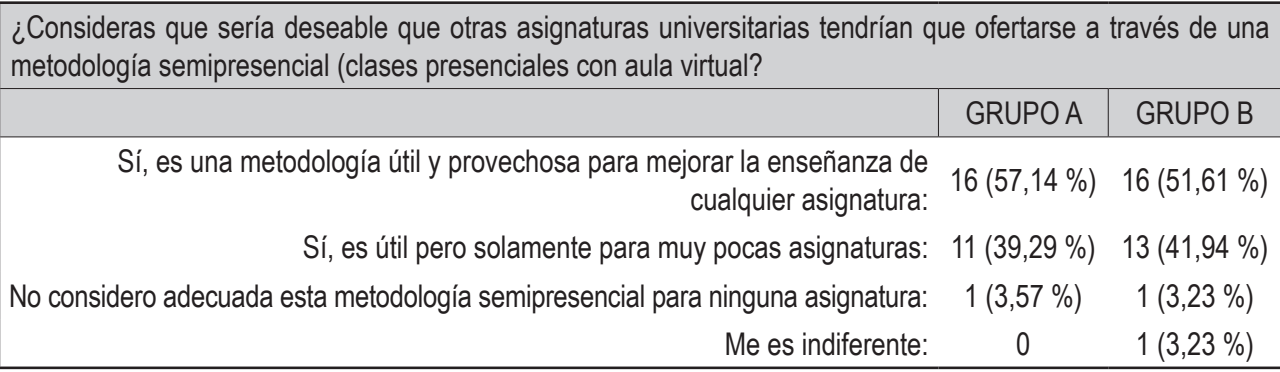

Tabla 12. Generalización de la experiencia a otras asignaturas

Para la mayoría del alumnado de los dos Grupos, ésta es la primera vez que estudian a través de un aula virtual (23 de los 28 alumnos del Grupo A, y 30 alumnos de los 32 del Grupo B). Mayoritariamente se conectan desde sus casas, aunque también hacen uso del aula de informática de la Facultad, y en menor medida, nos encontramos con alumnos-as que lo hacen desde un cibercafé. Al comparar este dato con el obtenido en el cuestionario inicial encontramos que el porcentaje de conexiones a través del aula de informática de la Facultad ha aumentado en los dos grupos (ver tabla 3 y 9), esto es debido a que en la organización de la asignatura se reserva tiempo para acceder a este servicio de la Facultad para facilitar el seguimiento de la asignatura.

Las valoraciones que hace el alumnado sobre esta experiencia, ver tabla 10, oscila entre los que consideran que han aprendido mucho y que les ha aportado cosas nuevas, valorándola de muy satisfactoria, y aquellos otros que consideran que la experiencia de cursar la asignatura a través del aula virtual, ha tenido algunos aspectos positivos pero otros no tanto, aunque la valoración global en ambos casos en positiva.

En cuanto a la utilidad de la experiencia, el alumnado la considera una metodología necesaria para mejorar la enseñanza (14 alumnos de los 28 del Grupo A, y 22 de los 32 alumnos del Grupo B), y útil para el aprendizaje (14 del Grupo A y 17 del Grupo B). No obstante, 9 alumnos del Grupo A y 13 del Grupo $\mathrm{B}$, consideran que la utilidad del aula virtual va a depender del profesor-a (tabla 11). A pesar de esta relación entre la utilidad de las aulas virtuales y el papel del profesorado, mayoritariamente consideran que en un futuro las asignaturas universitarias dispondrán de aulas virtuales. Y ello es así aunque su opinión sobre la generalización de este tipo de experiencia se encuentra dividida entre los que opinan que la metodología semipresencial es útil y provechosa para mejorar la enseñanza de cualquier asignatura, y los que la consideran útil solamente para algunas asignaturas (14 alumnos-as de los 28 del Grupo A, y 22 de los 32 alumnos-as del Grupo B marcan 
las dos opciones). Al comparar este dato con el obtenido en el cuestionario inicial (tabla 5 y tabla 12), encontramos que el porcentaje de estudiantes que consideran que se debería generalizar a todas las asignaturas ha aumentado después de cursar Tecnología Educativa, en ambos grupos, ya que opinan que esta metodología es útil y provechosa para cualquier asignatura.

\section{Segunda dimensión: Asignatura de Tecnología educativa}

\begin{tabular}{|r|c|c|}
\hline \multicolumn{3}{|c|}{ A la semana ¿cuántas horas aproximadamente has dedicado a trabajar esta asignatura } \\
(resúmenes, prácticas, conexión al aula, participar en el foro, etc.)? \\
\hline & GRUPO A & GRUPO B \\
\hline Menos de 3 horas semanales: & $14(50,00 \%)$ & $5(15,63 \%)$ \\
Entre 3 y 6 horas: & $12(42,86 \%)$ & $24(75,00 \%)$ \\
Entre 7 y 10 horas: & $2(7,14 \%)$ & $3(9,38 \%)$ \\
Más de 10 horas: & 0 & 0 \\
\hline
\end{tabular}

Tabla 13. Horas de trabajo

\begin{tabular}{|c|c|c|}
\hline & $\begin{array}{c}\text { ¿Has asistido regularmente a las } \\
\text { clases presenciales lunes? } \\
\text { GRUPO A }\end{array}$ & $\begin{array}{c}\text { ¿Has asistido regularmente a las } \\
\text { clases presenciales teóricas? } \\
\text { GRUPO B }\end{array}$ \\
\hline Sí: & $19(70,37 \%)$ & $25(78,13 \%)$ \\
No: & $8(29,63 \%)$ & $6(18,75 \%)$ \\
\hline
\end{tabular}

Tabla 14. Asistencia a las clases presenciales teóricas y prácticas

\begin{tabular}{|c|c|c|}
\hline & $\begin{array}{c}\text { ¿Has asistido a las clases prácticas en } \\
\text { el aula de informática? } \\
\text { GRUPO A }\end{array}$ & $\begin{array}{c}\text { ¿Has asistido regularmente a las } \\
\text { clases prácticas? } \\
\text { GRUPO B }\end{array}$ \\
\hline Sí: & $27(96,43 \%)$ & $32(100,00 \%)$ \\
No: & $1(3,57 \%)$ & 0 \\
\hline
\end{tabular}

Tabla 15. Asistencia a las clases presenciales teóricas y prácticas

\begin{tabular}{|c|c|c|}
\hline & GRUPOA & GRUPO B \\
\hline Muy satisfactorias. De lo mejor de la asignatura. Me han aportado mucho: & $10(37,04 \%)$ & $14(43,75 \%)$ \\
\hline Satisfactorias. A veces bien, otras regular, pero en conjunto aceptable: & $13(48,15 \%)$ & $14(43,75 \%)$ \\
\hline Indiferente. Han sido normales. Ni bien ni mal: & $4(14,81 \%)$ & $1(3,13 \%)$ \\
\hline Insatisfactoria. No me han aportado casi nada: & 0 & 0 \\
\hline
\end{tabular}

Tabla 16. Valoración de la asignatura 


\begin{tabular}{|r|c|c|}
\hline & $\begin{array}{c}\text { ¿Qué valoración realizas de las } \\
\text { clases prácticas de principio } \\
\text { del cuatrimestre en el aula de } \\
\text { informática? } \\
\text { GRUPO A }\end{array}$ & $\begin{array}{c}\text { ¿Qué valoración } \\
\text { realizas de las clases } \\
\text { prácticas? } \\
\text { GRUPO B }\end{array}$ \\
\hline $\begin{array}{r}\text { Muy satisfactorias. De lo mejor de la asignatura. } \\
\text { Me han aportado mucho: }\end{array}$ & $14(51,85 \%)$ & $17(53,13 \%)$ \\
$\begin{array}{r}\text { Satisfactorias. A veces bien, otras regular, pero } \\
\text { en conjunto aceptable: }\end{array}$ & $13(48,15 \%)$ & $15(46,88 \%)$ \\
Indiferente. Han sido normales. Ni bien ni mal: & 0 & 0 \\
Insatisfactoria. No me han aportado casi nada: & 0 & 0 \\
\hline
\end{tabular}

Tabla 17. Valoración de la asignatura

\begin{tabular}{|c|c|c|}
\hline \multicolumn{3}{|c|}{$\begin{array}{l}\text { Señala qué aspectos consideras como positivos o destacables de esta asignatura (puedes marcar varia } \\
\text { respuestas) }\end{array}$} \\
\hline & GRUPOA & GRUPO B \\
\hline El diseño del aula virtual utilizada: & $18(64,29 \%)$ & $19(59,38 \%)$ \\
\hline Los foros de debate: & $16(57,14 \%)$ & $15(46,88 \%)$ \\
\hline El calendario con las tareas: & $11(39,29 \%)$ & $16(50,00 \%)$ \\
\hline La sección de noticias del profesor: & $16(57,14 \%)$ & $16(50,00 \%)$ \\
\hline Las actividades prácticas propuestas: & $13(46,43 \%)$ & $16(50,00 \%)$ \\
\hline Las sesiones de clase presencial: & $4(14,29 \%)$ & $4(12,50 \%)$ \\
\hline El diario personal: & $4(14,29 \%)$ & $3(9,38 \%)$ \\
\hline La implicación del profesor en el desarrollo de la asignatura: & $16(57,14 \%)$ & $15(46,88 \%)$ \\
\hline La participación del alumnado en el desarrollo de la asignatura: & $16(57,14 \%)$ & $17(53,13 \%)$ \\
\hline Todos los anteriores: & $8(28,57 \%)$ & $3(9,38 \%)$ \\
\hline Ninguno de los anteriores: & 0 & 0 \\
\hline
\end{tabular}

Tabla 18. Valoración del aula virtual de la asignatura

\begin{tabular}{|c|c|c|}
\hline $\begin{array}{l}\text { Señala qué aspectos consideras como negativos o mal desarrollados de } \\
\text { alternativas) }\end{array}$ & a asignatur & es marcar varia \\
\hline & GRUPO A & GRUPO B \\
\hline El diseño del aula virtual utilizada: & 0 & $1(3,13 \%)$ \\
\hline Los foros de debate: & 0 & $1(3,13 \%)$ \\
\hline El calendario con las tareas: & $1(3,57 \%)$ & $1(3,13 \%)$ \\
\hline La sección de noticias del profesor: & 0 & $1(3,13 \%)$ \\
\hline Las actividades prácticas propuestas: & 0 & 0 \\
\hline Las sesiones de clase presencial: & $6(21,43 \%)$ & $4(12,50 \%)$ \\
\hline El diario personal: & $1(3,57 \%)$ & $3(9,38 \%)$ \\
\hline
\end{tabular}




\begin{tabular}{|r|c|c|} 
& GRUPO A & GRUPO B \\
La implicación del profesor en el desarrollo de la asignatura: & 0 & $1(3,13 \%)$ \\
La participación del alumnado en el desarrollo de la asignatura: & $3(10,71 \%)$ & $2(6,25 \%)$ \\
Todos los anteriores: & 0 & 0 \\
Ninguno de los anteriores: & $19(67,86 \%)$ & $21(65,63 \%)$ \\
\hline
\end{tabular}

Tabla 19. Valoración del aula virtual de la asignatura

\begin{tabular}{|c|c|c|}
\hline \multicolumn{3}{|c|}{$\begin{array}{l}\text { La metodología semipresencial utilizada en esta asignatura a través del aula virtual ¿qué te ha aportado? } \\
\text { (puedes marcar varias respuestas) }\end{array}$} \\
\hline & GRUPOA & GRUPO B \\
\hline Me ha dado más autonomía para desarrollar los trabajos y estudiar la teoría: & $16(57,14 \%)$ & $23(71,88 \%)$ \\
\hline Me ha permitido planificar mejor el tiempo dedicado a la asignatura: & $18(64,29 \%)$ & $16(50,00 \%)$ \\
\hline Me ha estimulado a buscar información y a elaborar mi propio conocimiento: & $12(42,86 \%)$ & $18(56,25 \%)$ \\
\hline Me ha motivado para implicarme más en el estudio de esta asignatura: & $9(32,14 \%)$ & $10(31,25 \%)$ \\
\hline Me ha gustado tanto que quisiera que la asignatura fuera totalmente a distancia: & $2(7,14 \%)$ & $2(6,25 \%)$ \\
\hline Me ha creado tantos problemas que no quiero volver a esta metodología: & 0 & $1(3,13 \%)$ \\
\hline No me ha aportado casi nada. Prefiero la metodología presencial: & 0 & 0 \\
\hline Me da lo mismo estudiar con una metodología presencial o semipresencial: & $1(3,57 \%)$ & 0 \\
\hline
\end{tabular}

Tabla 20. Valoración de la metodología semipresencial

\begin{tabular}{|r|c|c|}
\hline Valora la distribución del tiempo que se ha realizado en esta asignatura: \\
\hline & $\begin{array}{c}\text { GRUPO A } \\
(50 \% \text { presencial, } \\
50 \% \text { virtual })\end{array}$ & $\begin{array}{c}\text { GRUPO B } \\
(70 \% \text { presencial, } \\
30 \% \text { virtual })\end{array}$ \\
\hline Esta distribución me parece correcta y no la modificaría: & $15(55,56 \%)$ & $15(46,88 \%)$ \\
Aumentaría el tiempo dedicado a actividades virtuales: & $9(33,33 \%)$ & $14(43,75 \%)$ \\
Aumentaría el tiempo dedicado a actividades presenciales en clase: & $3(11,11 \%)$ & $1(3,13 \%)$ \\
Me es indiferente. No tengo una opinión formada: & 0 & $2(6,25 \%)$ \\
\hline
\end{tabular}

Tabla 21. Valoración de la metodología semipresencial

El ítem de respuesta abierta se dirige a la valoración sobre el aula virtual y la metodología semipresencial de la asignatura. Tras leer las aportaciones del alumnado, y ver que señalaban aspectos positivos o aspectos que eran susceptibles de mejoras, decidimos clasificarlas en dos grandes grupos, que denominamos de fortalezas y debilidades. No obstante, y ante la variedad de aspectos o elementos que señalaban, consideramos que resultaba más clarificador agrupar sus aportaciones atendiendo a los elementos a los que el alumnado hacía referencia. 
En esta segunda dimensión, centrada en la asignatura de Tecnología Educativa, nos encontramos con diferencias significativas entre los dos grupos. Así, y empezando por el análisis de la primera subdimensión, referida a los horas de trabajo, nos encontramos con que la mitad del alumnado del Grupo A ha dedicado menos de tres horas de trabajo a la semana, y de la otra mitad son mayoría el alumnado que ha dedicado entre tres y seis horas de trabajo a la asignatura, siendo sólo dos alumnos lo que dicen haber empleado entre siete y diez horas a la semana. Sin embargo, en el Grupo B nos encontramos con que mayoritariamente el alumnado señala que ha dedicado entre tres y seis horas semanales de trabajo a la asignatura, sólo cinco de los treinta y dos alumnos dice que ha trabajado menos de tres horas, y solamente tres señalan que han trabajado entre siete y diez horas a la semana (ver tabla 13). Quizás esta diferencia se deba a que a la hora de realizar el cálculo de las horas que dedicaron a trabajar en la asignatura incluyeron las horas de asistencia a clase, en este caso, el Grupo B tenía más horas de presencialidad que el Grupo A.

$\mathrm{Si}$ atendemos a la valoración que hace el alumnado de la asignatura, nos encontramos que en ambos grupos la valoración es positiva (ver tabla 18 y 19). Las diferencias entre grupo se encuentran en los aspectos o elementos que más valoran. De este modo nos encontramos que el alumnado del Grupo A lo que más valora, por orden de más a menos dentro de la valoración positiva, es el diseño del aula, los foros de debate, la sección de noticias del profesor, la implicación del profesor, la participación del alumnado, las actividades prácticas, y en menor medida, las sesiones de las clases presencial y el diario personal. En el Grupo B, el alumnado ha valorado en mayor medida el diseño del aula, la participación del alumnado, el calendario de tareas, la sección de noticias del profesor, las actividades prácticas, la implicación del profesor, y en menor medida, las sesiones de las clases presenciales y el diario.

Sobre la valoración que hacen de la metodología semipresencial, véase tabla 20, en líneas generales el alumnado de los dos grupos, destacan la autonomía, la planificación del trabajo, y la motivación para la búsqueda de la información y en la implicación en el estudio. De nuevo observamos diferencias entre los grupos en función del énfasis puesto en cada uno de los elementos o aspectos. Así, el alumnado del Grupo A destaca que la metodología semipresencial a través del aula virtual, les ha permitido planificar mejor el tiempo dedicado a la asignatura, en segundo lugar, la autonomía para el desarrollo de los trabajos y para el estudio, y en tercer y cuarto lugar, la motivación para la búsqueda de información y para la elaboración del conocimiento, y para la implicación en el estudio. El alumnado del Grupo B destaca, en primer lugar, la autonomía seguida de la motivación para la búsqueda de información y la elaboración 
del conocimiento, y en tercer lugar, la posibilidad de planificar mejor el tiempo, y por último, la motivación en la implicación en el estudio.

También, sobre esta subdimensión de la metodología semipresencial (ver tabla 20), nos encontramos con que en ambos grupos dos alumnos-as manifiestan la opinión de que la asignatura debería ser totalmente virtual. Aunque sobre este mismo aspecto, un alumno-a del Grupo B, dice que prefiere la metodología presencial, y un alumno-a del Grupo A, manifiesta que le es indiferente una u otra metodología.

En cuanto a la distribución de las horas presenciales y virtuales, se observan diferencias entre los dos grupos (ver tabla 21). Mientras que en el Grupo A, a la mitad del alumnado le parece correcta y adecuada la distribución del tiempo, y la otra mitad opina que aumentaría las actividades virtuales, siendo una minoría la que aumentaría el tiempo de las presenciales; en el Grupo B, la opinión del alumnado se divide entre los que les parece correcto y adecuado la distribución del tiempo entre la presencialidad y la virtualidad, y la de los que consideran que se debería aumentar el tiempo dedicado a las actividades virtuales.

A las diferencias sobre esta última subdimensión hay que añadirles que ambos grupos trabajaron con una distribución distinta de las horas presenciales y virtuales. Así, el Grupo A contó con un 50\% de presencialidad y un 50\% de virtualidad, y su opinión mayoritaria es que les parece correcta y adecuada dicha distribución del tiempo. Mientras que el Grupo B contó con un 70\% de presencialidad y un 30\% de virtual, encontrándose sus opiniones divididas entre los que opinan que es adecuada y los que aumentarían el tiempo de no presencialidad o virtualidad.

Las aportaciones que el alumnado realiza sobre el aula virtual y la metodología semipresencial desarrollada en la asignatura, recogidas en la pregunta abierta del cuestionario, vienen a ratificar los resultados expresados con anterioridad. Las opiniones del alumnado al respecto podríamos resumirlas diciendo que valoran muy positivamente tanto el aula virtual de la asignatura como la metodología semipresencial. La experiencia la califican de innovadora, interesante.... Destaca, en este sentido, sus opiniones sobre lo que han aprendido, donde hacen hincapié sobre todo en la autonomía en el aprendizaje, en la planificación y organización del estudio..., y señalan como debilidad el estudio de los contenidos teóricos de la asignatura. En esta misma línea, valoran también de muy satisfactoria el cómo han aprendido, es decir, la metodología semipresencial y el aula virtual. En este punto destaca las aportaciones y valoraciones que realizan de las interacciones entre el alumnado, y entre el profesorado y el alumnado, a través fundamentalmente de los foros, considerándolas una pieza clave el proceso de enseñanza y aprendizaje. 


\section{CONCLUSIONES FINALES}

Las conclusiones del estudio se han realizado a partir del análisis comparativo entre los resultados obtenidos en el cuestionario inicial y el final. En este sentido, presentamos estas conclusiones atendiendo a las relaciones de los resultados obtenidos en las distintas dimensiones utilizadas.

Cuando se inicia la experiencia de docencia virtual, el alumnado de la asignatura de Tecnología Educativa se encuentra alfabetizado como usuario de las tecnologías digitales. Se define como un usuario de Internet que utiliza tanto los recursos comunicativos como de información, aunque mayoritariamente lo utiliza en los contextos no formales de ocio para la comunicación entre sus iguales y búsqueda de elementos culturales como música y películas; sólo y ocasionalmente ha utilizado Internet en los contextos de educación formal, concretamente para buscar información en el proceso de aprendizaje de alguna asignatura. La gran mayoría del alumnado dispone de ordenadores personales que les ha facilitado este aprendizaje en el uso de las tecnologías digitales, y fundamentalmente de Internet.

Para el alumnado esta es la primera vez que experimentan un proceso de enseñanza y aprendizaje a través de un aula virtual y de una metodología semipresencial. Inician esta experiencia con unas expectativas altas sobre la docencia virtual, destacando la opinión de que les va a aportar autonomía y flexibilidad, y considerando que van a aprender más. Estas expectativas se confirman al final del proceso, tal y como se observa en los resultados del cuestionario final, donde se observa que mayoritariamente el alumnado valora la experiencia de muy satisfactoria. En este sentido destaca las opiniones que hace el alumnado sobre el aprendizaje en sus respuestas a la pregunta abierta, que lo define como un aprendizaje rico, que les ha aportado autonomía en el estudio y con el que han aprendido a planificar y organizar su estudio. Un ejemplo representativo de estas valoraciones es la opinión de un alumno-a que dice "que ha aprendido no sólo conocimientos nuevos sino también maneras de aprender”.

Donde el alumnado manifiesta un cambio de opinión es en las posibilidades de generalización de la experiencia a otras asignaturas. Así, mientras en el cuestionario inicial fueron pocos los alumnos-as que apuntaban esta posibilidad, considerando que resultaba adecuado para unas asignaturas pero para otras no; en el cuestionario final, la mitad del alumnado consideraba que en el futuro todas las asignaturas universitarias dispondrían de un aula virtual. 
Con respecto a la asignatura de Tecnología Educativa, las expectativas iniciales del alumnado se puede decir que se confirman al final del proceso. Tal y como se recoge en la tabla 1, el alumnado manifestaba que le gustaría conocer y aplicar las tecnologías en la educación, dominio de la alfabetización informática, y conocimiento y experiencias del aula virtual. Finalmente resta concluir que, en conjunto, las opiniones y valoraciones sobre el aula virtual y la metodología semipresencial desarrollada en la asignatura, se observa la alta satisfacción que manifiesta el alumnado sobre el proceso de la asignatura.

\section{NOTAS}

1 El campus virtual de la ULL y el dominio de Internet empleado fue creado originariamente en el año 2001 por el Laboratorio de Educación y Nuevas Tecnologías (EDULLAB) y gestionado por el mismo hasta el año 2004 a raíz de un proyecto impulsado por el Vicerrectorado de Servicios Generales. En 2003 dicho campus virtual fue financiado con una Cátedra Telefónica. Véase al respecto Area y otros (2002, 2003). En el sitio web de EDULLAB se puede encontrar más información sobre las acciones desarrolladas en dicho periodo http://www.edullab.org

2 El profesor del Grupo A tanto de las clases teóricas como prácticas fue Manuel Area. Las profesoras del Grupo B fueron Ana L. Sanabria (clases prácticas) y Miriam González (clases teóricas).

\section{REFERENCIAS BIBLIOGRÁFICAS}

Area, M.; otros (2003). Strategies for Information and Communication Technologies (ICT) Diffusion and Aplication in Higher Education: Case of La Laguna University Mendez-Vilas; Mesa; Mesa (eds.). Advances in TechnologyBased Education: Towards a Knowledge Based Society. Vol. I, 239-243.

Area, M.; otros (2004). Diseño y experimentación pedagógica de materiales didácticos distribuidos a través de la WWW. El caso de la asignatura de Tecnología Educativa. Qurriculum. Revista de Teoría, Investigación y Práctica Educativa, No 17, 141-160.

Boyle, T.; Bradley, C.; Chalk, P.; Jones, R.; Pickard, P. (2003). Using blended learning to improve student success rates in learning to program. Journal of
Educational Media, 28(2-3), 165-178.

Cebrián, M. (Coord) (2003). Enseñanza virtual para la innovación universitaria. Madrid: Narcea.

Colas, P.; De Pablos, J. (coords.) (2005). La universidad en la Unión Europea. El Espacio Europeo de Educación Superior y su impacto en la docencia. Málaga: Ediciones Aljibe.

Cox, G.; Carr, T.; Hall, M. (2004). Evaluating the use of synchronous communication in two blended courses. Journal of Computer Assisted Learning, 2O, 183-193.

Fonseca, Ma . C.; Aguaded J. I. (Dres.) (2007). Enseñar en la universidad. Experiencias y propuestas para la docencia universitaria. A Coruña: NetBiblo

Garrison, D.; Kanuka, H. (2004). Blended learning: Uncovering its transformative 
potential in higher education. Internet and Higher Education, $\mathrm{N}^{0}$ 7, $95-105$

Sangrá, A.; González, M. (coord) (2004). La transformación de las universidades a través de las TIC: discursos y prácticas. Barcelona: Editorial UOC.

Tejada, J.; Navío, A.; Ruíz, C. (2007). La didáctica en un entorno virtual universitario. Píxel-Bit, 30, 95-118.
Vignare, K.; otros (2005). Blended Learning Review of Research: An Annotative Bibliography, The ALN Conference Workshop on Blended Learning \& Higher Education [en línea] Disponible en: http://www.uic.edu/depts/oee/ blended/workshop/bibliography.pdf [consulta 2007, 20 de junio]

\section{PERFIL ACADÉMICO Y PROFESIONAL DE LA AUTORA}

Manuel Area Moreira es Dr. en Pedagogía y Catedrático de Didáctica y Organización Escolar en la Universidad de La Laguna. Dirige el Laboratorio de Educación y Nuevas Tecnologías, y es Presidente de la Red Universitaria de Tecnología Educativa.

E-mail: manarea@ull.es

Ana L. Sanabria Mesa, es Dra. en Pedagogía y Profesora de la Facultad de Educación en la Universidad de La Laguna. Sus publicaciones y proyectos de investigación se centran en la formación del profesorado, la innovación educativa y el uso educativo de las tecnologías de la información y comunicación.

E-mail: asanabri@ull.es

Mirian González Afonso, es Licenciada en Pedagogía y profesora ayudante del Dpto. de Didáctica e Investigación Educativa de la Universidad de La Laguna. Su campo de especialización es la Tutoría universitaria.

E-mail: mcglez@ull.es

DIRECCIÓN DE LOS AUTORES

Departamento de Didáctica y Organización

Escolar

Facultad de Educación. Módulo B

Campus Central.

Universidad de La Laguna

(Tenerife). España

Fechas de recepción del artículo: 17/10/07

Fechas aceptación del artículo: 14/01/08 